\title{
Re: Examination of the Significant Placebo Effect in the Treatment of Interstitial Cystitis/Bladder Pain Syndrome
}

\section{Bosch PC}

Palomar Medical Center, Department of Urology, CA, USA

Urology 2014;84:321-326. doi: 10.1016/j.urology.2014.04.011. Epub 2014 Jun 21.

\section{EDITORIAL COMMENT}

In this study, the efficacy of the treatment in the placebo arm of a study investigating the effects of subcutaneous adalimumab in patients with interstitial cystitis (IC) was evaluated. There are several controversies regarding the etiology and treatment of IC. In the studies of IC, although success rates were not very high in the treatment groups, success rates of $12 \%$ to $20 \%$ were observed in the placebo arms. The data in this study were extracted from a previous study of Bosch who investigated the effects of adalimumab in patients with IC in a placebo-controlled study (1). In the original study, 43 patients were grouped as treatment and placebo arms and the efficacy of adalimumab was investigated. The rate of patients, who had more than $50 \%$ global response rate, was $53 \%$ in the adalimumab arm and 50\% in the placebo arm. Although the difference was not statistically significant, the unexpected high response rate in the placebo arm stimulated the author to investigate the underlying factors in a second study. In this study, which investigated the effects of placebo in the treatment of IC, when the patients were questioned about the factors that made them feel better, being followed up closely by a physician, caring for their diet, increasing fluid intake, reduction of stress and regulation of eating habits were found as the most prominent factors. These findings were consistent with the results of the previous studies which showed the efficacy of conservative and behavioral treatment. Moreover, those treatment modalities sometimes can be more effective than certain medications. As a conclusion, when evaluating the studies regarding IC, one should keep in mind that a significant placebo effect can be observed. Additionally, the importance of behavioral treatment and counselling the patients accordingly should always be remembered.

\section{REFERENCE}

1. Bosch PC. A randomized, double-blind, placebo controlled trial of adalimumab for interstitial cystitis/bladder pain syndrome. J Urol 2014;191:77-82. 